\title{
Obtención De Alcohol A Partir De Remolacha
}

\author{
Brito Hanníbal \\ Docente Investigador, \\ Grupo de Investigación Ambiental y Desarrollo de la ESPOCH (GIADE), \\ Tapia Valeria \\ Usiña José
}

Docente Investigador Facultad de Ciencias,

\section{Guamán Horacio}

Puente César

Docente Investigador Facultad de Ciencias Pecuarias

\section{Patiño Teobaldo}

Mendoza Mery

Haro Carla

Colaborador Facultad de Ciencias,

Moreno Beatriz

Investigador ECUACERÁMICA

doi: 10.19044/esj.2016.v12n24p242 URL:http://dx.doi.org/10.19044/esj.2016.v12n24p242

\begin{abstract}
The preparation of beet alcohol by fermentation and distillation processes, has as a main objective is to obtain alcohol with purity in excess of $90 \%$, In order to accomplish this goal, initially we proceeded with a market sampling including Mayorista, La Condamine, La Merced, San Alfonso and La Esperanza markets in the city of Riobamba. Beet samples were taken in each of them and they were analyzed in the laboratory of industrial processes at the Polytechnic School of Chimborazo (ESPOCH). It was identified that the optimal beet for the fermentation process is the one collected at Mayorista market. It meets the highest percentage of solids with a 13,66 \% among the analyzed. Using the beet from Mayorista market, the amount of juice and water suitable for the preparation of $100 \mathrm{~L}$ of must was determined. Yeast dissolved in water and ammonium sulfate were to the mixture, after which, the reactor was sealed to start the fermentation process: Temperature and time variables were adjusted, later samples were taken every hour until achieving constant alcoholic strength, which was $12 \%$ in a time span of 12 hours. The ensuing product was fed to the distillation tower until reaching the $94 \%$ alcohol at a boiling temperature of $76{ }^{\circ} \mathrm{C}$. Optimum
\end{abstract}


process variables required in the fermentation process are: $\mathrm{T}=30^{\circ} \mathrm{C}, \mathrm{pH}=$ 4,3 and in the process of distillation; a boiling temperature of $76^{\circ} \mathrm{C}$ to reach an alcohol content of $94 \%$.

Keywords: Alcohol, Alcoholic fermentation, must, inoculation, saccharomyces cerevisiae, beer yeast, beet juiceProcess parameters

\section{Resumen}

La elaboración de alcohol de remolacha mediante los procesos de fermentación y destilación, tiene como objetivo principal la obtención de alcohol superior al 90\%, para lo cual, se procedió inicialmente con un muestreo en los mercados: Mayorista, La Condamine, La Merced, San Alfonso y La Esperanza de la ciudad de Riobamba, en los mismos que se tomaron muestras de remolacha que fueron analizadas en el laboratorio de Procesos Industriales de la Escuela Superior Politécnica de Chimborazo (ESPOCH), identificando que la remolacha óptima para el proceso de fermentación es la conseguida en el mercado Mayorista, misma que cumple con el mayor porcentaje de sólidos con un 13,66 \% entre los analizados. Con la remolacha procedente del mercado Mayorista, se procedió a determinar la cantidad de zumo y agua adecuada para la preparación de 100 L de mosto, a la mezcla se la adicionó levadura disuelta en agua y sulfato de amonio, luego de lo cual, se cerró herméticamente el reactor para iniciar el proceso de fermentación ajustando las variables de temperatura y tiempo, posteriormente se tomó muestras cada hora hasta alcanzar el grado alcohólico constante, mismo que fue de 12 \% en un lapso de tiempo de 12 horas, este producto así obtenido fue alimentado a la torre de destilación hasta llegar al 94\% de alcohol a una temperatura de ebullición de $76^{\circ} \mathrm{C}$. Las variables de proceso óptimas requeridas en el proceso de fermentación son: $\mathrm{T}$ de $30{ }^{\circ} \mathrm{C}, \mathrm{pH}$ de 4,3 y en el proceso de destilación $\mathrm{T}$ de ebullición de $76{ }^{\circ} \mathrm{C}$ para llegar a un grado alcohólico de $94 \%$.

Palabras Clave: Alcohol, Fermentación Alcohólica, mosto, inoculación, saccharomyces cerevisiae, levadura de cerveza, zumo de remolacha, parámetros del proceso

\section{Introducción}

Para la elaboración de alcohol a partir de la remolacha, se parte de un proceso de fermentación alcohólica (Fermentación alcohólica, H.J. Vázquez1 y O. Dacosta 2011), misma que tiene como objetivo la transformación de un mosto de remolacha (preparados de extractos y zumos de frutas y hortalizas) (Agro negocios Ecuador 2015) en alcohol por medio de la acción microbiana que utilizan las reacciones de fermentación como vía metabólica para 
obtener energía y materia indispensable para su desarrollo y su posterior destilación para llegar a un grado alcohólico superior al 90\%. Esta fermentación se la puede realizar sin la utilización de instrumentos y equipos de precisión diseñados para obtener la máxima eficiencia, pero esto conlleva a mayores tiempos de fermentación, baja calidad de los productos, posibles pérdidas de lotes de producción por la acción de fermentación láctica (Elaboración de cerveza utilizando cebada y yuca 2010) y productos tóxicos que no hacen posible un proceso viable y de calidad. (Doran 1998).

La fermentación alcohólica se realizó en un reactor de camisa que fue diseñado y construido en el laboratorio de procesos industriales de la Escuela de Ingeniería Química de la Escuela Superior Politécnica de Chimborazo, para aprovechar al máximo la acción de los microorganismos sobre los azúcares del mosto, transformándolos en alcohol, biomasa y demás productos de fermentación, para ello se consideró las condiciones ideales en las cuales los microorganismos fermentativos se desarrollaron en su máxima expresión (Guarnizo, A. 2008). El factor principal en el proceso de fermentación fue mantener las variables como la temperatura y la agitación ideal, para que los microorganismos se desarrollen y consuman a tasas elevadas los sustratos transformándolos en alcohol y sus subproductos (Universidad de Sevilla, 2014).

Para este propósito se analizó inicialmente el tipo de remolacha más adecuada para los procesos fermentativos, esto se lo hace por un muestreo a nivel de los mercados La Condamine, La Merced, San Alfonso y La Esperanza de la ciudad de Riobamba, determinando el mayor porcentaje de sólidos en la remolacha 13,66 \%. (La remolacha fue adquirida en el Mercado Mayorista, ubicado en la ciudad de Riobamba en la provincia de Chimborazo). Se buscó un lote de remolacha que provenga del mismo sistema de cultivo, presente con el mismo tiempo de cosecha, es decir, se procuró obtener una materia prima lo más homogénea e idónea posible para el sistema de fermentación. Una vez determinadas las cantidades de zumo de remolacha y agua se procedió a la medición de la velocidad de reacción, para lo cual, se preparó 100 L de mosto, se añadió 200 g de levadura, previamente disuelta en $500 \mathrm{~mL}$ de agua a $32{ }^{\circ} \mathrm{C}$ (hidratada y activada), $200 \mathrm{~g}$ de sulfato de amonio y se procedió a fermentar en a una temperatura de $30{ }^{\circ} \mathrm{C}$ por un lapso de 12 horas. Cada hora se tomó una muestra del mosto de $20 \mathrm{~mL}$, al mismo que se determina el grado alcohólico obtenido, siendo del $12 \%$, además se determinó las variables óptimas de operación como son temperatura de fermentación $30^{\circ} \mathrm{C}$; $\mathrm{pH} 4,3$; tiempo y agitación cada $10 \mathrm{~min}$ durante 2 min y a 20 RPM.

El producto obtenido en el proceso de fermentación con el $12 \%$ de alcohol se alimenta a la torre de destilación hasta obtener un grado alcohólico (López E, 2013) de 94 \%, a una temperatura de ebullición de76 
${ }^{\circ} \mathrm{C}$, de esta manera se ha cumplido con el objetivo planteado al inicio de la investigación.

\section{Metodología}

Este trabajo de investigación se realizó en el laboratorio de procesos industriales de la Escuela de Ingeniería Química de la Facultad de Ciencias de la Escuela Superior Politécnica de Chimborazo, el cual, se orientó a la obtención de una bebida alcohólica a partir de la remolacha y posteriormente a la destilación para lograr un alto grado alcohólico por destilación fraccionada $\left(94^{\circ} \mathrm{GL}\right)$.

Para el desarrollo de esta investigación se empleó un muestreo de selección previa de la materia prima basándose en el tamaño, indicado en la norma INEN 1832 “Hortalizas Frescas Remolacha”, así como la variación de costos y disponibilidad; se eligió trabajar con la remolacha del Mercado Mayorista, la misma que proviene del Cantón Chambo, debido a que, al realizar el análisis tiene la mayor concentración de de sólidos totales (13,66\%), este valor representa la cantidad de sacarosa presente en el tubérculo (Laboratorio de Procesos Industriales, ESPOCH, 2015).

Con la remolacha seleccionada se procedió a la extracción de zumo de acuerdo a la formulación específica descrita en la técnica para la fermentación de alcoholes que se ubica en el laboratorio de procesos industriales. El zumo fue filtrado para eliminar partículas pequeñas de sólidos en suspensión, luego se procedió a la carga del zumo en el reactor, se preparó el mosto añadiendo agua y posteriormente se inoculó la levadura de cerveza, la cual, previamente fue activada con agua a la temperatura establecida, posteriormente se le agrego sulfato de amonio el cual va a actuar como nutriente para los microorganismos, se cerró el fermentador, iniciando el proceso de fermentación.

Cada hora se recolectó una muestra para la determinación de sólidos totales, grados Brix, $\mathrm{pH}$ (las muestras deben recolectarse en tubos de ensayo con tapa de volumen mayor a $20 \mathrm{~mL}$ ), el equipo se apaga automáticamente cuando se mantiene constante los grados Brix y el $\mathrm{pH}$, este proceso se demora 12 horas por carga (Lab. Procesos Industriales, 2015).

Estos ensayos de laboratorio y pruebas en el fermentador se realizaron con el fin de determinar y optimizar las variables de proceso como temperatura, grados Brix, $\mathrm{pH}$, velocidad de reacción, tiempo de residencia y que el equipo cumpla con su mayor eficiencia, (Lab. Procesos Ind. 2015).

Una vez terminado el proceso de fermentación se procede a la descarga del producto y a la vez se carga el mismo en el destilador, se enciende el caldero y se empieza con el proceso de destilación, al final del mismo se obtuvo etanol a una concentración de $94{ }^{\circ} \mathrm{GL}$. 
CUADRO 1 Propuesta del proceso de obtención de alcohol a partir de remolacha (Equipo de fermentación Lab. Procesos Industriales ESPOCH)

ETAPA

Preparación del
zumo
Preparación del
mosto
Inoculado del
mosto

Fermentación del mosto
Rectificación del producto

CONDICIONES

Extraer 86 L del zumo de remolacha

azucarera Beta vulgaris $L$. El zumo debe ser preparado por licuefacción de remolacha previamente lavada y cortada los tallos y cualquier imperfección, posteriormente tamizada para retener la fracción de sólidos de tamaño considerable. Todas las operaciones se deben realizar en condiciones asépticas y con materiales previamente lavadas para asegurar la inocuidad del producto.

Adicionar 14 litros de agua a los 86 litros de zumo de remolacha para obtener la concentración de sólidos deseado, el agua debe estar previamente tratada para asegurar la inocuidad del producto. La mezcla se puede realizar en el equipo, previamente al lavado del mismo.

A la mezcla se procede a adicionar la levadura activada (200 g de levadura sólida disuelta en agua a $30{ }^{\circ} \mathrm{C}$ hasta eliminar la totalidad de grumos) más $200 \mathrm{~g}$ de sulfato de amonio como agente bio estimulante.

El proceso de fermentación del mosto se lo realiza con el equipo cerrado con la

tapa hermética procurando que los empaques no se dañen, ajustar los tornillos de sujeción evitando el sobresfuerzo de los mismos para limitar los daños internos por la dilatación. A continuación se enciende el equipo y se configura el sistema automático para que la temperatura se mantenga en $30^{\circ} \mathrm{C}$, con esto se logra fermentar el $80 \%$ de la sacarosa en un tiempo de fermentación de 12 horas por batch.

Para incrementar el grado alcohólico y la pureza del producto de la fermentación se debe realizar una destilación fraccionada hasta el grado alcohólico deseado.
Concentración de sólidos 13,66 \%

$30^{\circ} \mathrm{C}$

$20^{\circ} \mathrm{C}$

\author{
En función del \\ grado alcohólico \\ deseado en el rectificado
}

Fuente: BRITO H. / et al, Laboratorio Procesos industriales, ESPOCH, 2015. 


\section{Resultados Y Discusión}

En la tabla 1 se indica que la remolacha con más alto contenido de sólidos totales se obtuvo en el Mercado Mayorista de Riobamba, la misma que proviene del Cantón Chambo.

TABLA 1 Contenido de sólidos en las diferentes remolachas muestreadas en los mercados de Riobamba

\begin{tabular}{|c|c|c|c|c|c|c|}
\hline Mercados & $\begin{array}{c}\text { PESO } \\
\text { CRISOL } \\
\text { VACÍO Y } \\
\text { TARADO } \\
\text { (g) }\end{array}$ & $\begin{array}{c}\text { PESO } \\
\text { CRISOL } \\
\text { MÁS } \\
\text { MUESTRA } \\
(\mathbf{g})\end{array}$ & $\begin{array}{c}\text { PESO } \\
\text { MUESTRA } \\
(\mathbf{g})\end{array}$ & $\begin{array}{c}\text { PESO } \\
\text { CRISOL } \\
\text { FINAL } \\
(\mathbf{g})\end{array}$ & $\begin{array}{c}\text { PESO } \\
\text { SOLIDOS, } \\
(\mathrm{g})\end{array}$ & $\begin{array}{c}\text { CONTENIDO } \\
\text { SOLIDOS, \% }\end{array}$ \\
\hline Mayorista & 24,95 & 34,25 & 9,66 & 25,91 & 1,32 & 13,66 \\
\hline $\begin{array}{c}\text { La } \\
\text { Condamine }\end{array}$ & 81,37 & 91,66 & 10,29 & 82,59 & 1,22 & 11,85 \\
\hline La Merced & 31,35 & 41,94 & 10,59 & 32,64 & 1,29 & 12,18 \\
\hline $\begin{array}{c}\text { San } \\
\text { Alfonso }\end{array}$ & 31,96 & 42,35 & 10,39 & 33,17 & 1,21 & 11,64 \\
\hline $\begin{array}{c}\text { La } \\
\text { Esperanza }\end{array}$ & 31,12 & 41,15 & 10,03 & 32,15 & 1,03 & 10,26 \\
\hline
\end{tabular}

Fuente: BRITO H. / et al, Laboratorio Procesos industriales, ESPOCH, 2015.

TABLA 2 Contendido de carbohidratos en la remolacha

\begin{tabular}{|c|c|c|}
\hline No. & CARBOHIDRATO & $\begin{array}{c}\text { CANTIDAD } \\
\text { g/100g remolacha }\end{array}$ \\
\hline 1 & Azúcares totales & 8,38 \\
\hline 2 & Fructosa & 0,25 \\
\hline 3 & Galactosa & 0,27 \\
\hline 4 & Glucosa & 0 \\
\hline 5 & Lactosa & 0 \\
\hline 6 & Maltosa & 0 \\
\hline 7 & Oligosacáridos & 7,86 \\
\hline 8 & Sacarosa & 0,00 \\
\hline 9 & Almidón & 0 \\
\hline 10 & Almidón resistente & 0,80 \\
\hline 11 & Celulosa & 0,31 \\
\hline 12 & Lignina & 0,94 \\
\hline 13 & Polisacáridos no celulósicos insolubles & 0,48 \\
\hline 14 & Polisacáridos no celulósicos solubles & . \\
\hline
\end{tabular}

Fuente: BRITO H. / et al, Laboratorio Procesos industriales, ESPOCH, 2015.

Para determinar la concentración del mosto en función de la concentración de sacarosa (representada por el contenido de sólidos totales) se utilizó los datos descritos en tabla 3. 
TABLA 3 Densidad de la solución de sacarosa en función al porcentaje de sacarosa

\begin{tabular}{|c|c|c|}
\hline No. & CONCENTRACIÓN SACAROSA (\%) & DENSIDAD SOLUCIÓN $(\mathrm{g} / \mathrm{mL})$ \\
\hline 1 & 5 & 1,017 \\
\hline 2 & 6 & 1,0204 \\
\hline 3 & 7 & 1,0238 \\
\hline 4 & 8 & 1,0272 \\
\hline 5 & 9 & 1,0306 \\
\hline 6 & 10 & 1,034 \\
\hline 7 & 11 & 1,0374 \\
\hline 8 & 12 & 1,0408 \\
\hline 9 & 13 & 1,0442 \\
\hline 10 & 14 & 1,0476 \\
\hline 11 & 15 & 1,051 \\
\hline
\end{tabular}

FUENTE: GUARNIZO, 2008.

TABLA 4 Formulación de agua requerida en la preparación del mosto

\begin{tabular}{cccc} 
ENSAYO & PESO REMOLACHA (g) & PORCENTAJE AGUA (\%) & $\begin{array}{c}\text { CANTIDAD } \\
\text { AGUA (L) }\end{array}$ \\
\hline 1 & 50 & 12 & 0,006 \\
2 & 50 & 14 & 0,007 \\
3 & 50 & 16 & 0,008 \\
4 & 50 & 18 & 0,009 \\
5 & 50 & 20 & 0,01
\end{tabular}

Fuente: BRITO H. / et al, Laboratorio Procesos industriales, ESPOCH, 2015.

TABLA 5 Contenido de sólidos para determinar el agua requerida en la preparación del mosto

\begin{tabular}{|c|c|c|c|c|c|c|}
\hline ENSAYO & $\begin{array}{c}\text { PESO } \\
\text { CRISOL } \\
\text { VACÍO Y } \\
\text { TARADO } \\
(\mathrm{g})\end{array}$ & $\begin{array}{c}\text { PESO } \\
\text { CRISOL } \\
\text { CON LA } \\
\text { MUESTRA } \\
(\mathrm{g})\end{array}$ & $\begin{array}{c}\text { PESO } \\
\text { MUESTRA } \\
(\mathrm{g})\end{array}$ & $\begin{array}{c}\text { PESO } \\
\text { CRISOL } \\
\text { FINAL } \\
(\mathrm{g})\end{array}$ & $\begin{array}{c}\text { PESO } \\
\text { SOLIDOS } \\
(\mathrm{g})\end{array}$ & $\begin{array}{c}\text { CONTENIDO } \\
\text { SOLIDOS (\%) }\end{array}$ \\
\hline 1 & 24,95 & 34,25 & 9,66 & 25,91 & 1,32 & 13,66 \\
\hline 2 & 81,37 & 91,66 & 10,29 & 82,59 & 1,22 & 11,85 \\
\hline 3 & 31,35 & 41,94 & 10,59 & 32,64 & 1,29 & 12,18 \\
\hline 4 & 31,96 & 42,35 & 10,39 & 33,17 & 1,21 & 11,64 \\
\hline 5 & 31,12 & 41,15 & 10,03 & 32,15 & 1,03 & 10,26 \\
\hline
\end{tabular}

Fuente: BRITO H. / et al, Laboratorio Procesos industriales, ESPOCH, 2015.

TABLA 6 Resultados de la determinación de la velocidad de reacción de la fermentación

\begin{tabular}{|c|c|c|c|c|}
\hline $\begin{array}{c}\text { TIEMP } \\
\text { O }(\mathrm{H})\end{array}$ & $\begin{array}{c}\text { CONCENT } \\
\text { RACIÓN } \\
\text { SOLIDOS } \\
\text { TOTALES } \\
(\%)\end{array}$ & $\begin{array}{c}\text { DENSIDAD } \\
\text { MOSTO } \\
(\mathrm{g} / \mathrm{mL})\end{array}$ & $\begin{array}{c}\text { CONCENTRACIÓ } \\
\text { N SACAROSA } \\
(\mathrm{g} / \mathrm{mL})\end{array}$ & $\begin{array}{c}\text { CONCENTRACIÓN } \\
\text { SACAROSA (mol/L) }\end{array}$ \\
\hline 0 & 13,2100 & 1,044914 & 0,13803314 & 0,40326372 \\
\hline 1 & 13,1800 & 1,044812 & 0,13770622 & 0,40230863 \\
\hline 2 & 13,1400 & 1,044676 & 0,13727043 & 0,40103546 \\
\hline 3 & 13,1189 & 1,04460426 & 0,13704059 & 0,40036398 \\
\hline 4 & 13,0800 & 1,044472 & 0,13661694 & 0,39912629 \\
\hline 5 & 13,0465 & 1,0443581 & 0,13625218 & 0,39806065 \\
\hline
\end{tabular}




\begin{tabular}{|c|c|c|c|c|}
\hline 6 & 13,0500 & 1,04437 & 0,13629029 & 0,39817197 \\
\hline 7 & 12,9800 & 1,044132 & 0,13552833 & 0,39594593 \\
\hline 8 & 12,9544 & 1,04404496 & 0,13524976 & 0,39513208 \\
\hline 9 & 12,9437 & 1,04400858 & 0,13513334 & 0,39479196 \\
\hline 10 & 12,9040 & 1,0438736 & 0,13470145 & 0,39353019 \\
\hline 11 & 12,8735 & 1,0437699 & 0,13436972 & 0,39256104 \\
\hline 12 & 12,8316 & 1,04362744 & 0,1339141 & 0,39122995 \\
\hline
\end{tabular}

Fuente: BRITO H. / et al, Laboratorio Procesos industriales, ESPOCH, 2015.

TABLA 7 Corrientes de alimentación y descarga en el equipo

\begin{tabular}{ccccc} 
No. & TIPO DE & COMPONENTE & FLUJO (g/h) & $\begin{array}{c}\text { FLUJO } \\
(\mathrm{Kg} / \mathrm{h})\end{array}$ \\
& CORRIENTE & $\mathrm{C}_{12} \mathrm{H}_{22} \mathrm{O}_{11}$ & 342,29265 & 0,34 \\
1 & Alimentación & $\mathrm{O}_{2}$ & 306,5926 & 0,31 \\
& & $\mathrm{NH}_{3}$ & 2,0162 & 0,00 \\
& & $\mathrm{CH}_{1,70} \mathrm{O}_{0,46} \mathrm{~N}_{0,17}$ & 49,062 & 0,05 \\
2 & \multirow{2}{*}{ Descarga } & $\mathrm{CO}_{2}$ & 435,66588 & 0,44 \\
& & $\mathrm{C}_{2} \mathrm{H}_{5} \mathrm{OH}$ & 169,079 & 0,17
\end{tabular}

Fuente: BRITO H. / et al, Laboratorio Procesos industriales, ESPOCH, 2015.

TABLA 8 Datos comparativos entre la velocidad de reacción experimental y la que registro el equipo en la validación de la fermentación de la remolacha

\begin{tabular}{|c|c|c|c|c|}
\hline $\begin{array}{c}\text { TIEMPO } \\
(\mathrm{h})\end{array}$ & \begin{tabular}{c}
$\boldsymbol{C}_{\boldsymbol{S T}} \begin{array}{c}\text { EXPERIMENTAL } \\
(\mathrm{mol} / \mathrm{L})\end{array}$ \\
\hline 1
\end{tabular} $\boldsymbol{r}_{\boldsymbol{f}} \begin{array}{c}\text { EXPERIMENTAL } \\
(\mathrm{mol} / \mathrm{L} * \mathrm{~h})\end{array}$ & $\begin{array}{c}\boldsymbol{C}_{\boldsymbol{S} \boldsymbol{T}} \text { EXPERIMENTAL } \\
(\mathrm{mol} / \mathrm{L})\end{array}$ & $\begin{array}{c}\boldsymbol{r}_{\boldsymbol{f}} \text { EQUIPO } \\
(\mathrm{mol} / \mathrm{L} * \mathrm{~h})\end{array}$ \\
\hline 2 & 0,40326372 & - & 0,24232242 & - \\
\hline 3 & 0,40230863 & 0,00032692 & 0,23453557 & 0,00266536 \\
\hline 4 & 0,40103546 & 0,0004358 & 0,22255096 & 0,00410222 \\
\hline 5 & 0,40036398 & 0,00022984 & 0,21825616 & 0,00147007 \\
\hline 6 & 0,39912629 & 0,00042365 & 0,21883092 & $-0,00019673$ \\
\hline 7 & 0,39806065 & 0,00036476 & 0,21702978 & 0,00061651 \\
\hline 8 & 0,39817197 & $3,8105 \mathrm{E}-05$ & 0,21430269 & 0,00093346 \\
\hline 9 & 0,39594593 & 0,00076195 & 0,21377092 & 0,00018202 \\
\hline 10 & 0,39513208 & 0,00027857 & 0,2148844 & $-0,00038113$ \\
\hline 11 & 0,39479196 & 0,00011642 & 0,21150031 & 0,00115834 \\
\hline 12 & 0,39353019 & 0,00043189 & 0,21019453 & 0,00044696 \\
\hline 13 & 0,39256104 & 0,00033173 & 0,20968806 & 0,00017336 \\
\hline
\end{tabular}

Fuente: BRITO H. / et al, Laboratorio Procesos industriales, ESPOCH, 2015.

Según (Los alimentos, 2014) la mayor cantidad de carbohidratos presente en la remolacha es la sacarosa con un contenido de 7,86 g en relación a los demás, por lo que, se tomó la remolacha con mayor contenido de sólidos para el proceso de fermentación.

En cuanto a la temperatura se determinó un valor de $30{ }^{\circ} \mathrm{C}$, que en este caso es el parámetro óptimo, para la fermentación alcohólica, desarrollando adecuadamente las levaduras, de acuerdo a los requerimientos 
iniciales de la investigación. Por otro lado, el pH óptimo identificado en el mosto fue del 4,3; con este valor las levaduras actuaron eficientemente, en el proceso de conversión de la sacarosa en metanol, a valores diferentes de $\mathrm{pH}$ se inhibió el crecimiento de los microorganismos. El porcentaje de alcohol producido en el proceso de conversión de sacarosa en etanol fue del $12 \%$. El alcohol obtenido por el proceso de fermentación, fue destilado, llegando a una concentración de $94{ }^{\circ} \mathrm{GL}$ de alcohol etílico.

Por otro lado, al comparar la velocidad de fermentación experimental con la velocidad de reacción a la cual opera el equipo se evidencia que el mismo transforma las materias primas e insumos en productos y residuos a una mayor velocidad aplicando las mismas condiciones de operación como se ilustra en la tabla 8. En vista a que el equipo supera la velocidad de reacción esperada, el tiempo de residencia necesario para trasformar el 80\% de la sacarosa es inferior al tiempo necesario para transformar en la misma proporción de sacarosa del mosto procedente de la remolacha que se determinó experimentalmente.

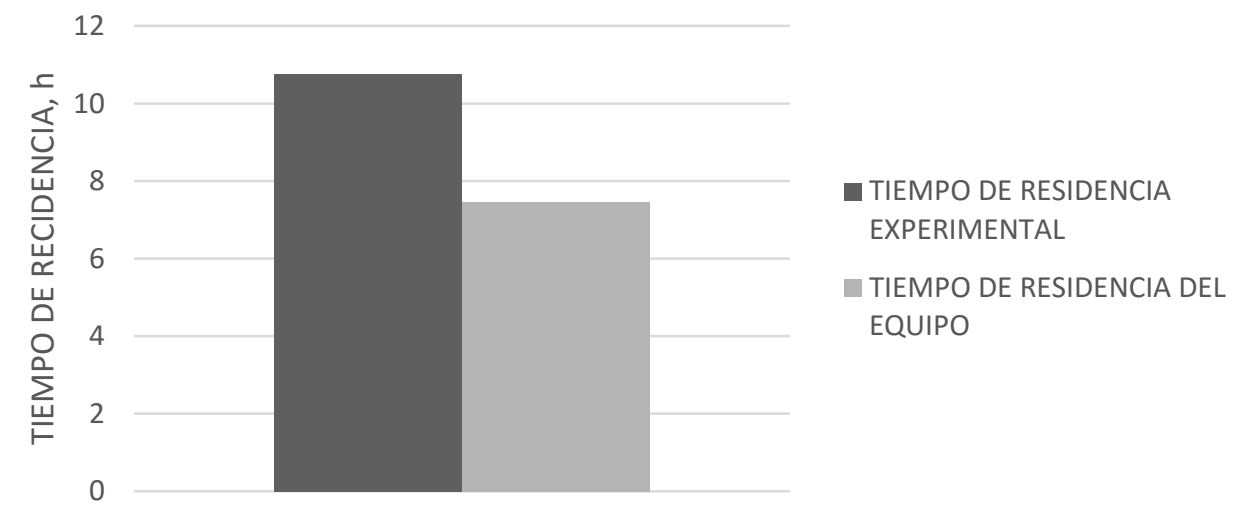

GRÁFICO 1 Tiempo de residencia experimental frente al tiempo de residencia del equipo necesario para transformar el $80 \%$ de la sacarosa del mosto

El predominio en la capacidad de operación que presenta el equipo en la fermentación del mosto de remolacha frente a los datos experimentales fueron principalmente a que el equipo presentó una mayor homogeneidad en la temperatura de operación, la cual, debió mantenerse lo más próxima a 30 ${ }^{\circ} \mathrm{C}$ para asegurar la fermentación a un régimen continuo, lo que se traduce en una mayor producción, mayor productibilidad de los resultados, menor tiempo de operación, menos consumo energético y menor control frente a la obtención del producto de manera experimental como se muestra en el gráfico 1. 


\section{Conclusion}

$\checkmark \quad$ Al realizar el muestreo y previo análisis se identificó que la remolacha del Mercado Mayorista de Riobamba, la misma que proviene del Cantón Chambo tiene la mayor cantidad de sacarosa.

$\checkmark \quad$ Para la preparación de $100 \mathrm{~L}$ de mosto se determinó que se debe adicionar $14 \%$ de agua.

$\checkmark \quad$ Se determinó que al controlar las variables de operación el pH óptimo en el mosto fue del 4,3 ya que las levaduras actúan de una forma eficiente.

$\checkmark \quad$ Se obtiene una rápida y mayor conversión de la sacarosa en etanol a una temperatura $30^{\circ} \mathrm{C}$ y agitación de $20 \mathrm{RPM}$.

$\checkmark \quad$ Al finalizar el proceso se obtuvo, como producto final de la fermentación y su posterior destilación un alcohol con una concentración del $94 \%$ en volumen a una temperatura de ebullición de $76^{\circ} \mathrm{C}$.

\section{References:}

RENDUELES, M.; Díaz, M. (2014). "Biotecnología industrial". Arbor, 190 (768): a155.

DEL MORAL Sandra / et al, (2014), Revista Iberoamericana de Ciencias, Aspectos relevantes del uso de enzimas en la industria de los alimentos.

Cortes-Lopez N.G., Montor-Antonio, J.J., Olvera-Carranza, C., Pena-Castro, J.M., del Moral, S. (2014). Metagenómica: una ventana de oportunidad a nuevos genes y genomas microbianos. Revista Iberoamericana de Ciencias.

SÁNCHEZ Juan (2013), La Química del Color en los Alimentos, Química Viva, Buenos Aires Argentina, Vol. 12.

Binod, P., Palkhiwala, P., Gaikaiwari, R., Nampoothiri, K.M., Duggal, A., Dey Kand Pandey, A. (2013). Industrial Enzymes - Present status and future perspectives for India. J Sci. Ind. Res. India. 72: 271-286.

CHATEAUNEUF Rolando, El consumo de una bebida a base de remolacha, zanahoria y manzana curaría el cáncer y tendría otros importantes efectos en el organismo, 2012.

Li, S., Yang, X., Yang, S., Zhu, M., Wang, X. (2012). Technology prospecting on enzymes: Application, marketing and engineering. CSBJ. 2:3.

PADÍN Carmiña / DÍAZ Mario, 2012, Fermentación alcohólica del lactosuero por Kluyveromyces marxianus y solventes orgánicos como extractantes, Revista de la Sociedad Venezolana de Microbiología, Scielo, Venezuela.

Diario oficial de la federación. (México). 16 de julio de 2012. Acuerdo por el que se determinan los aditivos y coadyuvantes en alimentos, bebidas y suplementos alimenticios, su uso y disposiciones sanitarias Company, Novozymes. (2011). The Novozymes Report. 
Chang A., / et al (2011), Hydrogen production by the anaerobic fermentation from acid hydrolyzed rice straw hydrolysate. Int. J. Hydrogen Energy 36(21): 14280-14288.

Jiang, C., Wu, L.L., Zhao, G.C., Shen, P.H., Jin, K., Hao, Z.Y., et al. (2010). Identification and characterization of a novel fumarase gene by metagenome expression cloning from marine microorganisms. Microb. Cell Fact. 9:1-19. Tasse, L., Bercovici, J., Pizzut-Serin, S., Robe, P., Tap, J., Klopp, C., et al. (2010). Functional metagenomics to mine the human gut microbiome for dietary fiber catabolic enzymes. Genome Res. 20:1605-1612.

Buitrón G \& Carvajal C (2010) Biohydrogen production from Tequila vinasses in an anaerobic sequencing batch reactor: Effect of initial substrate concentration, temperature and hydraulic retention time, Biores, Technol, 101: 9071-9077.

Han, J., Morton, J.D., Bekhit, A.E.D., Sedcole, J.R. (2009). Prerigor infusion with kiwifruit juice improves lamb tenderness. Meat Sci. 82:324-330.

FERREYRA María / DÍAZ Fernández, 2009, Fermentación alcohólica de jugo de naranja con S. cerevisiae, Ciencia, Docencia y Tecnología $N^{0} 39$, Año XX, noviembre de 2009.

Health Canada. Recuperado el 16 de enero de 2015 en http://www.hcsc.gc.ca/fn-an/securit/addit/food_enzymes-eng.php.

CARREÓN R., / et al (2009), Etanol carburante, BioTecnología 13(3): 79102.

Guarnizo, A. (2008). Quimica General. Armendia: Elizcom.

HADER I. / et al, 2008, Ethanol production from cassava starch using the process strategy simultaneous saccharification - fermentation, Vitae vol.15 no.2 Medellín July/Dec. 2008.

PADÍN Carmiña, 2006, Efecto de la concentración inicial del lactosuero sobre la fermentación alcohólica con Kluyveromyces fragilis, Rev. Soc. Ven. Microbiol. v.26 n.1 Caracas jun. 2006.

SÁNCHEZ Óscar / CARDONA Carlos, 2005, Producción biotecnológica de alcohol carburante I: obtención a partir de diferentes materias primas, Scielo, Venezuela.

LEVENSPIEL, O. (2004). Ingeniería de las reacciones químicas. Mexico D.F.: Limusa. 